\title{
Mme. Yvonne Choquet-Bruhat
}

honoring 70 years of her work and major accomplishments in the area at the crossroads of Mathematics and Physics, applying results from the analysis of partial differential equations and differential geometry to problems in Physics.

For her major achievements Mme. Yvonne Choquet-Bruhat received many honors and, in 1979, she became the first woman admitted to the French Academy of Sciences.

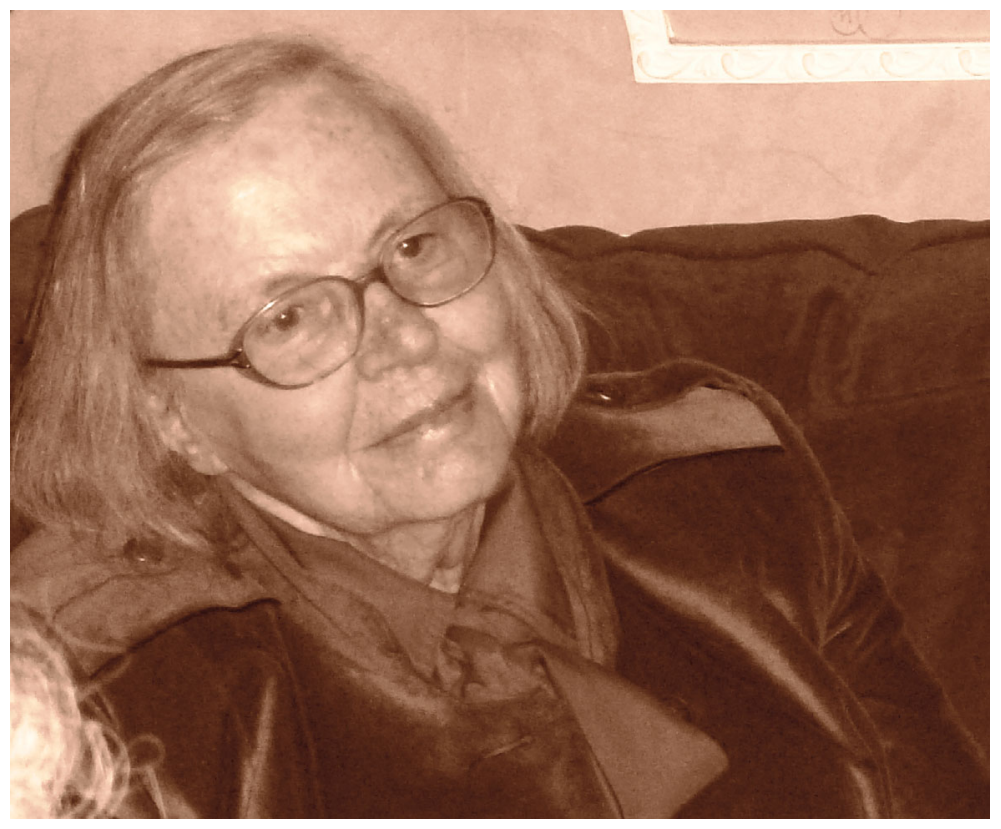

